\title{
L'improvisation et le jeu masqué dans la formation de l'acteur
}

\author{
Guy Freixe \\ Université Franche-Comté, Besançon, France. \\ Email: guy.freixe@gmail.com
}

Cet article traite sur la pédagogie de lacteur dont les techniques du masque et de l'improvisation sont les fondements et les instruments privilégiés pour établir le jeu. Cette formation a comme influence la filiation Jacques Copeau Jacques Lecoq - Ariane Mnouchkine, dont les travaux privilégient le jeu, où l'homme agit et pense avec tout son corps, pouvant inventer avec le masque un théâtre poétique.

Mots-clés

Masque. Improvisation. Jeu.
Este artigo trata sobre a pedagogia do ator, onde as técnicas da máscara e da improvisação são as bases e instrumentos privilegiados para estabelecer o jogo. Esta formação tem como influência a filiação Jacques Copeau Jacques Lecoq - Ariane Mnouchkine, cujos trabalhos privilegiam o jogo onde o homem age e pensa com o seu corpo, podendo inventar, com a máscara, um teatro poético.

Palavras-chave

Máscara. Improvisação. Jogo. 
«Vous avez une double responsabilité : recevoir, et avoir la générosité d'essayer de transmettre à celui qui est derrière vous. " 1

Je voudrais aborder cette question de la transmission de façon plus personnelle que ce que j'ai pu faire jusqu'à présent. Je me réfèrerai donc à des moments particuliers de mon travail de formateur autour du jeu masqué tout en le situant dans la filiation dans laquelle il s'inscrit $^{2}$. J'ai donné mon premier stage, pour acteurs professionnels, en juin 1987, à Montréal, pour trois semaines. Je venais de « sortir » du Théâtre du Soleil (je n'ai pas écrit « quitter », car je savais alors que j'allais poursuivre d'une autre manière mon compagnonnage avec la troupe; d'ailleurs j'étais venu à ce stage avec les masques du Soleil qui m'avaient été prêtés par Erhard Stiefel, le sculpteur de masque associé à cette compagnie). J'étais entré au Théâtre du Soleil quelques années plus tôt, en juin 1981, juste après ma formation à l'École Jacques Lecoq. Pour donner ce stage, je pouvais donc m'appuyer sur ces deux expériences. Ce que je fis. Dans la préparation de mes cours, plus que les continuités ce sont les différences qui m'apparurent alors entre ce qui n'était chez Lecoq qu'un travail de formation, où nous ne faisions que passer quelques minutes en scène, et la recherche d'un jeu visant l'incarnation d'un personnage telle que je venais de l'éprouver dans le cycle des Shakespeare et le Sihanouk ${ }^{3}$. L'école d'un côté, la création

1 Ariane Mnouchkine, introduction, choix et présentation des textes par Béatrice Picon-Vallin, Actes Sud-Papiers, coll. " Mettre en scène », Arles, 2009, p. 99.

2 Cf. mon ouvrage La Filiation Copeau-Lecoq-Mnouchkine, une lignée du jeu de l'acteur, préface de Georges Banu, L'Entretemps, coll. « Les voies de l'acteur », Paris, 2014.

3 Les Shakespeare (Richard II, La Nuit des Rois, Henry IV 1ère partie), traduction et mise en scène d'Ariane Mnouchkine, création du Théâtre du Soleil, 1981-1984 ; L'Histoire terri- de l'autre. J'étais à la recherche d'une complémentarité entre l'analyse du mouvement et les exercices d'improvisation de Lecoq et l'intensité des états dramatiques demandée par Mnouchkine. Aujourd'hui, ce que j'ai découvert auprès de ces deux maîtres, reliés par l'esprit d'une même filiation remontant à l'aventure du théâtre du Vieux-Colombier de Jacques Copeau (1879-1949), dont je vais tenter d'esquisser les principes fondamentaux pour le jeu de l'acteur, nourrit toujours mon enseignement ${ }^{4}$.

\section{L'École de l'improvisation}

Deux écoles françaises ont particulièrement marqué la pédagogie du jeu de l'acteur au XXe siècle: I'École du Vieux-Colombier de Jacques Copeau (1921-1924), et l'École Internationale de Théâtre de Jacques Lecoq qui reprend, en les structurant et les approfondissant, les intuitions premières du « maître-rêveur $»^{5}$. Cette pédagogie du jeu de l'acteur, tournant le dos à l'analyse psychologique et à l'interprétation des textes, repose avant tout sur cette idée maîtresse que l'homme reçoit le monde par son corps et pense et agit avec son corps. À l'approche introspective, elle oppose une visée phénoménologique. L'acteur ne part pas de lui mais du monde. Il vise l'impersonnalité pour mieux recevoir les dynamiques du vivant

ble mais inachevée de Norodom Sihanouk roi du Cambodge, d'Hélène Cixous, création du Théâtre du Soleil, 1985.

4 J'enseigne actuellement le jeu masqué et l'improvisation dans trois Écoles nationales supérieures d'art dramatique : L'ENSATT de Lyon, l'École de la Comédie de Saint-Étienne et l'ESNAM de Charleville-Mézières. Je donne aussi régulièrement des stages de formation professionnelle de longue durée agréés AFDAS et par la Région lle-de-France.

5 L'expression est d'Ariane Mnouchkine, dans « Raconter l'histoire de notre temps ", L'Âge d'or, première ébauche, Stock, coll. "Théâtre ouvert », Paris, 1975, p. 17. 
et en rejouer les formes multiples et les structures profondes. Le masque est l'instrument privilégié de cette pédagogie de la distance qui passe par l'effacement de la subjectivité. La choralité en est le pendant.

Copeau a été un semeur de graines. Un initiateur et un éveilleur. II a toujours pensé avant même de fonder, il y a de cela tout juste cent ans, le Théâtre du Vieux-Colombier - que la rénovation urgente du théâtre devait passer en premier par l'acteur. Et qu'il fallait débarrasser la scène et le jeu des tricheries et faussetés accumulées depuis des décennies. C'est du côté de l'enfance, de l'instinct de jeu de l'enfant, que Copeau va chercher les fondements de sa pédagogie. Avait-il en tête l'aphorisme de Baudelaire : «Le génie, c'est l'enfance retrouvée à volonté "? Le fait est que Copeau attachait la plus grande importance au jeu des enfant et avait jugé bon de ne pas envoyer les siens à l'école, comme nous l'apprend sa fille Marie-Hélène Dasté, pour qu'ils tirent de leurs jeux seul toute leur instruction : «Nous étions habités par une espèce d'invention perpétuelle, encouragée par mon père, et qui d'ailleurs pour lui était comme la loi, le secret de l'éducation d'un acteur: lui faire garder son esprit d'enfance, lui faire retrouver toujours cette faculté d'émerveillement et d'étonnement qui est celle des enfants ${ }^{6}$.

Copeau voulait préserver chez l'acteur cette faculté de poésie et de créativité, cet état de jeu fait de disponibilité, d'invention, d'attention et d'imagination, et c'est avec de jeunes enfants de 10 à 14 ans qu'il va commencer, pendant

6 Rencontre avec Marie-Hélène Dasté, Catherine Dasté, Christophe Allwright : une famille d'acteurs, rencontre organisée le 19 mars 1993 dans le cade des Ateliers de formation permanente du Théâtre du Campagnol, éd. Théâtre du Campagnol - Centre Dramatique National de Corbeil-Essonnes, VHS. la guerre, à mener ses premiers exercices pédagogiques pour se préparer à l'ouverture de son École. En proposant un travail autour des fables de La Fontaine, il se montre dès le début vigilant à ce que les enfants entrent dans les personnages par le corps: non pas seulement en « signifiant » l'animal pour qu'il soit reconnaissable, mais en le mimant intérieurement, en recherchant par exemple la démarche silencieuse et déliée du chat pour qu'apparaisse son caractere ${ }^{7}$... Copeau veut que ce soit par le corps et une imagination toute physique que l'enfant se lance en premier dans le jeu dramatique. II se méfie des mots et de l'analyse psychologique des personnages de la fable, comme si la rationalité du langage éloignait l'enfant du mimétisme premier et de la spontanéité créatrice. Copeau se dit enchanté par ces premiers exercices sur l'improvisation à partir des animaux et il est conscient qu'ils vont orienter sa recherche future : «Cette mine d'observation, écrit-il dans son Cahier, et l'expérience de ces exercices seront d'un grand secours à l'artiste pour la représentation plus ou moins forcée et caricaturée des personnages de la comédie ${ }^{8}$.

Copeau cherche à déclencher chez l'enfant un jeu libre, inventif, personnel qui le "transporte" dans l'imaginaire. Il attachera à ce mouvement libératoire la plus grande importance par la suite. Mais pour cela, il faut que l'enfant entre totalement dans son jeu, qu'il joue vraiment, qu'il soit $a k u^{9}$, c'est-à-dire qu'il quitte

\footnotetext{
7 Jacques COPEAU, Registres VI : L'École du Vieux-Colombier, textes établis, présentés et annotés par Claude Sicard, Gallimard NRF, coll. "Pratiques du théâtre », Paris, 2000, p. 99.
}

8 Idem, p. 101.

9 « $A k u$ », c'est-à-dire engagé entièrement dans le jeu. Voici ce que Copeau écrit dans son Journal à propos du jeu de ses propres enfants : « Depuis assez longtemps j'entendais les enfants dans leurs jeux se servir de ce terme : aku (prononcez 
la réalité pour vivre dans l'imaginaire. Et pour cela, il lui faut la plus grande liberté dans le jeu, sans inféodation à l'écrit : "Se défier surtout de ce qui nous rapproche de la littérature. Fuir aux antipodes. Pas de rapport ni de communication. II ne s'agit pas d'improviser d'après, mais d'improviser tout court. Pas d'intermédiaire. L'improvisateur vaut ce qu'il vaut. Et tant vaut l'improvisation... ${ }^{10}$. Copeau se rend compte alors qu'il y a deux écoles possibles, qui s'opposent quant à la nature du jeu recherché: l'école de l'improvisation et l'école de l'interprétation. Dans l'improvisation, l'acteur est confronté avant tout au présent et à l'instant partagé avec le partenaire et le public. Dans l'interprétation, qui part du texte dramatique, il doit médiatiser avec l'épaisseur sémantique du langage. Copeau opte pour l'improvisation. II s'en ouvre rapidement à ses deux compagnons de théâtre, Charles Dullin et Louis Jouvet, qui sont au front. Leur correspondance, de décembre 1915 à avril 1916, tourne autour de cette formidable révolution qu'est la découverte du jeu improvisé et des perspectives nouvelles ouvertes par celle-ci. En janvier 1916, Copeau franchit une nouvelle étape. II découvre avec enthousiasme les improvisations clownesques des Fratellini. II écrit à sa femme : « J'ai été cinq fois au Cirque revoir les mêmes clowns»"11.

acou). "Être aku", "tu n'es pas aku". J'ai l'explication. Aku est l'abréviation du mot danois akurat qui veut dire : tout à fait, complètement. Être aku, dans le jeu, c'est se confondre tout à fait avec le personnage, la chose, l'événement qu'on veut représenter, c'est faire corps avec son jeu, le prendre pour la réalité, en éprouver les sentiments, en mimer les gestes avec continuité sans permettre aux autres, aux parents, ni à soi-même, la notion d'une feinte provisoire. " Jacques COPEAU, Journal 1901-1915, t. I, lundi 4 mars 1912, Seghers, Paris, 1991, p. 557.

10 Note de Jacques COPEAU sur la lettre de Louis JOUVET du 10 janvier 1916, in Registres VI : L'École du Vieux-Colombier, op. cit., p. 110.

11 Jacques Copeau, Cahier "La Comédie improvisée ", Registres III : Les Registres du Vieux Colombier I, textes re-
Il admire leurs lazzi, leur jeu sans cesse renouvelé avec le public, et « la grâce alerte de leurs corps entraînés ${ }^{12}$ qui leur permet de trouver une transposition des sentiments par une écriture poétique du geste. En s'entretenant avec eux, il note que " c'est l'habitude de travailler ensemble qui est le secret de leur verve ${ }^{13}$, et il est frappé par la justesse de la définition d'un des principes de leur art : «Le mouvement, le rythme et la précision ${ }^{14}$.Il établit alors un lien entre la force de ces improvisations et cet espace sans décor de la piste qui permet à l'acteur de déployer le maximum de son pouvoir suggestif. Il note dans son Journal: « Médrano. Le cirque, par l'agencement et les proportions de son architecture réalise, dans une certaine mesure, cette aire vide du théâtre sur laquelle mon imagination joue à l'aise. Petite proportion des personnages et leur complète liberté d'allure, avec le vide autour d'eux ${ }^{15}$. Au vide du plateau, cet espace de tous les possibles, Copeau rêve d'accorder le vide intérieur de l'acteur. Un vide qui fasse vibrer l'être en le reliant à l'authenticité de son engagement. Un vide mystérieusement plein. Dès lors, il renonce à emplir l'acteur d'informations et d'explications sur le caractère de son personnage et préfère faire appel à la puissance mystérieuse de ce vide pour que les ailes de son imagination se déploient librement. La fascination exercée par le masque à l'École du Vieux-Colombier se comprend mieux quand on la relie à cette pré-

cueillis et établis par Marie-Hélène Dasté et Suzanne Maistre Saint-Denis, Gallimard NRF, coll. « Pratique du théâtre », Paris, 1979 , p. 319.

12 Ibid.

13 ld., p. 322.

14 Ibid.

15 Jacques Copeau, Journal - 1916-1948, t. II, 1ier septembre 1916, Seghers, Paris, 1991, p. 22-23. 
occupation d'atteindre ce vide matriciel cher à Copeau. Le masque n'a pas été porté à l'École seulement pour gommer les tics et les habitudes, il n'a pas été l'instrument d'une technique; il est apparu plutôt comme l'instrument privilégié de ce "transport" sacré qui fonde, pour Copeau, le jeu de l'acteur.

\section{En premier, le corps}

Jacques Lecoq (1921-1999) a oeuvré dans la même voie que celle initiée par Copeau, et son école repose sur les trois mêmes piliers: l'improvisation, l'analyse du mouvement et le travail collectif des élèves eux-mêmes, qu'il nommera « auto-cours ». Lecoq a reçu cet héritage pendant les deux années où il a fait partie de la troupe de Jean Dasté, Les Comédiens de Grenoble. Par la suite, il a su donner force et cohérence aux fondamentaux de cette recherche en faisant du masque, de la commedia dell'arte et du chœur tragique les bases de son enseignement qu'il a mis en place avec Giorgio Strehler à l'École du Piccolo Teatro de Milan, en 1951, et qu'il a continué en ouvrant à Paris, en 1956, son École Internationale de Théâtre. Lecoq a trouvé dans les travaux de Marcel Jousse ${ }^{16}$ un appui théorique à cette pédagogie « mimo-dynamique » telle que Copeau et Suzanne Bing l'avaient initiée dans le creuset de leur école. Car c'est là qu'eurent lieu pour la première fois les exercices mimées où, sous le masque, les élèves jouèrent à être " le vent du matin qui se réveille », « deux arbres qui s'agitent douce-

16 Marcel Jousse (1886-1961), élève de Marcel Mauss, a donné des cours d'Anthropologie à la Sorbonne, de 1931 à 1957. Cet enseignement a donné lieu à la publication posthume de trois ouvrages : L'Anthropologie du geste ; La Manducation de la parole ; Le Parlant, la parole et le souffle, aujourd'hui rassemblés dans L'Anthropologie du geste, Gallimard, coll. « Tel », Paris, 2008. ment », " l'oiseau », " le cerf » et « la nuit qui se réveille doucement et devient l'Aurore ${ }^{17}$. Lecoq fit de ces «identifications » aux dynamiques de la nature et aux animaux le socle de son enseignement. Sous le masque « neutre », cet autre nom qu'il donnera au masque « noble » de Copeau, il mit en place un « voyage » pour ouvrir l'élève à plus grand que soi.

Ces identifications marquent dans le processus pédagogique de son école une étape importante car elles correspondent à une plongée dans l'invisible. Ce retrait de soi apporté par la mise du masque est fondamental car il facilite le ressenti intérieur. II donne la permission de s'aventurer, par mimétisme, dans "l'espace du dedans“. II protège et unifie : sous ce masque, quelles que soient les cultures et les couleurs de peau différentes, tous les élèves se ressemblent et il ne reste plus que la vibration essentielle de l'être présent dans l'espace. « L'expérience m'a prouvé, écrit Lecoq, qu’il se passait avec ce masque des choses fondamentales qui en ont fait le point central de ma pédagogie ${ }^{18}$. Ce masque apprend à agrandir les gestes et les attitudes ; l'élève devient alors plus sensible à cet état d'équilibre lié à l'économie du mouvement : "Parce qu'il connaît l'équilibre, remarque Lecoq, l'acteur exprimera bien mieux les déséquilibres des personnages ou des conflits ${ }^{19}$. Mais le masque neutre apporte aussi une mutation, car l'acteur est amené à "essentialiser" le geste, c'est-à-dire à le simplifier, en le densifiant de l'intérieur. Du coup, les grandes lignes de fond restent et les petits

17 Notes de Marie-Hélène Daste in Jacques Copeau, « Cahier d'exercice de Maiène », Registres VI : L'École du Vieux-Colombier, op. cit., p. 350.

18 Jacques Lecoq, Le Corps poétique, en collaboration avec Jean-Gabriel Carasso et Jean-Claude Lallias, Actes Sud-Papiers/ANRAT, Arles, 1999 p. 47.

19 Ibid. 
détails s'estompent. Après les éléments de la nature: l'eau, le vent, la terre, le feu, les identifications continuent avec les matières: le bois, le papier, le métal, le verre, le caoutchouc, l'huile, le velours ou la soie... et c'est autant d'ouvertures à des circuits nouveaux de sensations, de rythmes, de formes et d'énergie. Lecoq souhaite ainsi que les élèves « entrent dans le goût des choses, exactement comme un gourmet peut reconnaître les différences subtiles entre des saveurs ${ }^{20}$. Le rythme de la forêt, la lumière solaire, la montagne que l'on gravit, la pente que l'on descend pour regarder le crépuscule dans la plaine... l'élève doit d'abord en retrouver l'impression dans son corps, avant d'en donner une expression. L'identification « mimo-dynamique » permet ainsi de faire ressurgir des gestes oubliés. L'élève retrouve alors ce que le corps a gardé en mémoire de toutes ses expériences sensibles, et c'est de ces strates enfouies que naissent les élans profonds de sa créativité. La nature, pour Lecoq, est notre premier langage et, comme il aimait à le dire souvent, «le corps, se souvient ${ }^{21}$.

Depuis, le masque neutre est devenu un outil essentiel dans la pédagogie de l'acteur et il est enseigné dans de nombreuses écoles de par le monde. Ce masque, ai-je pu écrire dans mon ouvrage sur Les Utopies du masque, appelé "noble" (Copeau), "impersonnel" (Dullin), "sublime" (Decroux), "pur" (Sartori), "transparent" (Strehler), avant que Lecoq lui trouve le qualificatif qui lui est resté : “"neutre", est le masque de l'exercice ${ }^{22}$. II est devenu aujourd'hui une référence incontournable. Dans ma pédagogie,

20 Jacques LECOQ, Le Corps poétique, op. cit., p. 55.

21 Idem., p. 56.

22 Guy FREIXE, Les Utopies du masque sur les scènes européennes $d u X X e$ siècle, L'Entretemps, coll. « Les voies de l'acteur », Montpellier, 2010, p. 272. outre sa faculté à éveiller la conscience corporelle, élargir le vocabulaire gestuel de l'acteur, dé-psychologiser le jeu et trouver une écriture du mouvement transposée dans l'espace, je I'utilise beaucoup pour travailler cette entité purement dramatique qu'est le chœur. Là aussi il s'agit de passer par un effacement du moi de l'acteur pour faire vivre un corps collectif et se sensibiliser à l'écoute des autres et à la dynamique du mouvement dans l'espace.

\section{- Découvrir les principes du jeu masqué}

L'importance du masque, aujourd'hui, est plus à chercher dans la pédagogie du jeu de l'acteur que dans l'usage, somme toute très limité, qui lui est accordé dans les spectacles. Dans cet espace de l'apprentissage, le masque occupe une place privilégiée qui passe par des stages, des ateliers, des laboratoires de recherche. En plus du masque neutre, qui n'est pas à proprement parler un masque de jeu, divers types de masque y sont utilisés, mais principalement ceux de deux traditions de théâtre privilégiant la comédie : les demi-masques en cuir de la commedia dell'arte et ceux en bois du Topeng balinais. Les stages organisés par Ariane Mnouchkine, au Théâtre du Soleil, proposent un travail qui mélange ces deux traditions masquées. Depuis plus de trente ans, dans ces stages-rencontres, elle ne travaille qu'à partir des masques. Ce sont eux, aime-t-elle à dire, qui «font tout le travail » : ils servent à révéler les tempéraments, aviver les imaginations, faire naître le théâtre, et par conséquent permettre des naissances d'acteur. Si le masque est pour Mnouchkine la « 
discipline de base ${ }^{23}$ de l'acteur, c'est qu'il lui apprend avant toute chose à ne pas chercher à se montrer lui-même, mais au contraire à accepter de se dépouiller le plus possible pour accueillir l'"étranger" et l'"étrange" en soi, ce qui n'arrive qu'à travers ce fameux « état » dont Mnouchkine parle souvent, et qui n'est qu'un autre nom du « transport » cher à Copeau.

Comment travailler le masque ? Y-a-il une technique spécifique pour aborder ce style de jeu ? Pour Mnouchkine, avant toute chose, il est nécessaire que l'acteur se prépare à le recevoir. Car chaque masque a une histoire et une " âme complète "24. Quand l'acteur se trouve devant une rangée de masques issus de traditions différentes, il a devant lui I'humanité dans sa quintessence, présente dans toutes ces faces colorées venues de la diversité des cultures du monde, et qui sont autant de facettes de notre moi profond. Pour pouvoir recevoir cette humanité contenue dans ces masques, l'acteur devra apprendre à être le plus disponible possible, aussi « vide » que le plateau du théâtre demandé par ce type de jeu.

Ensuite, le processus du travail est essentiel. Car travailler le masque sollicite une pratique collective du théâtre. En effet, de la même manière que le masque n'appartient pas à l'acteur, le travail qui est mené sous le masque appartient à tous les participants. On ne peut en effet comprendre ce type de jeu qu'en passant alternativement d'un côté à l'autre de la scène, et en observant attentivement le même masque joué par plusieurs acteurs. L'individu s'efface donc devant le masque qui est l'objet

23 Cf. Ariane MNOUCHKINE, "Le masque, une discipline de base ", in Le masque. Du rite au théâtre, textes réunis et présentés par Odette Aslan et Denis Bablet, CNRS, Paris, 1985, p. 231-234.

24 Selon la terminologie d'Ariane Mnouchkine. d'une recherche collective. C'est une des raisons pour lesquelles ce type de jeu se retrouve principalement dans les aventures théâtrales privilégiant le jeu collectif.

Le masque impose ses contraintes: il oblige l'acteur à se dégager des gestes quotidiens et des attitudes réalistes. La transposition devient nécessaire car sous le masque l'acteur doit "traduire" par le corps l'état émotionnel de son personnage. Les signes qu'il utilise doivent être très précis, car le masque agit comme une loupe. Même s'il ne bouge qu'un orteil, ou un doigt de la main, ce mouvement prend tout à coup une force inouïe. Le jeu masqué permet la prise de conscience de l'articulation nécessaire du mouvement, en étant attentif au démarrage et à la fin du geste, et en ponctuant rythmiquement ses attitudes pour que la "phrase corporelle" soit lisible. Le masque ouvre la voie métaphorique, celle où l'acteur montre par des signes physiques ce qui l'affecte en dedans, comme s'il donnait à voir les symptômes de son affection psychique. En cela il est la voie souveraine de l'art de l'acteur qui, comme le dit Mnouchkine, " est d'abord une autopsie et ensuite une symptomatique. Ou plutôt une symptomatique, et ensuite une autopsie $»^{25}$. Cette nécessité de transposer l'état intérieur dans une forme corporelle stricte développe l'imagination, considéré comme le "muscle" principal de l'acteur. Les contraintes du masque ouvrent donc à une liberté d'expression inattendue et à une écriture maîtrisée des signes, conjuguant élan émotionnel et mise en forme corporelle rigoureuse.

Dès que l'acteur entre en scène, il faut que tout son corps raconte avec la plus grande

25 « Une troupe, si elle est digne de ce nom, est toujours une école ", Entretien avec Ariane Mnouchkine, in Guy FREIXE, La Filiation Copeau-Lecoq-Mnouchkine, une lignée du jeu de l'acteur, op. cit., p. 268. 
clarté possible le personnage, son état, et ses raisons d'agir. Le mot ne doit pas venir trop tôt pour que l'acteur exprime dans et par son corps ce qu'il y a avant les mots, et qui constitue le moteur essentiel de l'action dramatique. Ariane Mnouchkine aime enseigner ce b.a.-ba du jeu, revenir sans cesse sur les fondamentaux de ce qu'elle aime appeler « le » théâtre. Elle a le goût de l'apprentissage, des commencements et, par dessus tout, celui de donner naissance à l'acteur. Elle définit souvent son travail comme celui d'une sage-femme. Elle dirige le jeu de l'acteur avec une telle maîtrise que ses stages, attendus par des centaines de jeunes comédiennes et comédiens, sont de grandes leçons de théâtre. Avec beaucoup d'humour, de la ferveur, un engagement sans limite, elle apprend alors aux jeunes acteurs, à travers le jeu masqué, cette écriture de l'acteur passant par le langage du corps. Par son regard, son rire, ses indications, elle l'aide à préciser sa silhouette, sa démarche, mieux dessiner ses attitudes, éliminer tel geste parasite, et surtout intensifier son moteur de jeu, qu'elle appelle parfois " sa petite musique intérieure $»^{26}$. Dès le premier pas sur le plateau, elle a tout vu : le corps mal placé, le rythme flou, l'agitation fébrile. Elle dit alors simplement « Non. Reprends ! ». Et dès qu'elle repère un élément juste dans le jeu sur lequel elle peut prendre appui, elle demande alors à l'acteur de recommencer; et avec une patience infinie, dix fois, vingt fois de suite, elle peut retravailler le même détail de jeu pour que le geste s'affine et traduise, en des variations infinies, la passion du personnage. Elle cherche à lui faire comprendre par-là ce qu'écrire veut dire, quand tout le corps est parfaitement dessiné, de l'or- teil jusqu'à la pointe des cheveux. C'est par la précision d'une attitude, le soin d'un détail, ce qu'elle appelle le « petit », que l'acteur atteint le « grand ». Et pour cela, il ne doit jamais jouer deux choses à la fois. II faut qu'il écrive lettre après lettre. Ariane demande à l'acteur que ses gestes soient purs, attentionnés, que le pourtour de son corps soit soigneusement sculpté, avec des angles vifs, et des arrêts qui viennent rythmer le mouvement...

Cette écriture des signes corporels, à laquelle le jeu masqué initie, n'est jamais travaillée comme une fin en soi. Elle doit venir extérioriser ce qu'il y a de plus intime, la vérité même des sentiments. La grande loi, bien mystérieuse, réside dans ce passage entre l'intérieur et l'extérieur, entre l'état et sa mise en forme par le corps. Ariane Mnouchkine reste vigilante à ce que celle-ci ne devienne pas une déformation, et c'est pour cela qu'elle rejette la caricature comme étant une facilité, voire une duperie. Tout l'art de l'acteur consiste justement à ce que son écriture vienne extérioriser l'intérieur sans tomber dans l'extériorité. Et c'est justement cela que les grands maîtres orientaux enseignent. Si Mnouchkine s'en remet au masque pour guider l'acteur dans le jeu c'est que, bien porté, il agit dans les deux sens : il permet de préciser la forme extérieure, tout en ouvrant intérieurement de nouveaux circuits sensibles et créatifs. II sensibilise l'acteur à la "graphie" du corps, tout en servant de tremplin à son imaginaire.

26 Toujours selon la terminologie d'Ariane Mnouchkine. 
Ouvrir les possibles avec des masques de différentes traditions

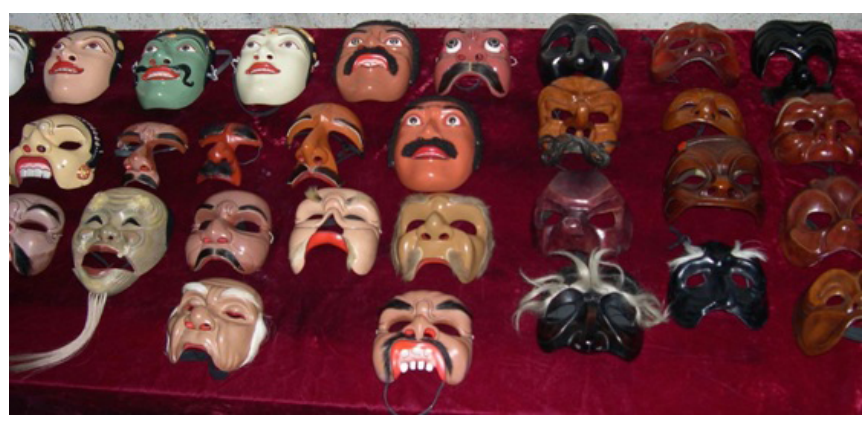

Font: Guy Freixe.

Ce sont ces fondamentaux du jeu masqué que je cherche à mon tour à transmettre dans mon enseignement théâtral. Celui-ci fait appel, comme on vient de le voir, à l'imagination de l'acteur et trouve son terrain de prédilection dans l'improvisation. Mais on peut improviser à partir de supports très différents : une thématique générale (la peste, la guerre, les nouvelles odyssées des migrants contemporains, le naufrage et le débarquement sur une île, etc.) ; un simple canevas (avec situation, personnages, développement d'un conflit dramatique) ; ou en prenant appui sur un texte. Depuis des années, j'aime recourir à ce type de confrontation, qui ne va pas sans tensions, entre les contraintes du masque et les exigences du texte.

Le masque est un outil magistral dans la mesure où il oblige l'acteur à trouver une forme. Mais il faut le reconnaître, le masque est aussi tyrannique. Il demande à ce qu'on lui obéisse. Le maquillage supporte fort bien que l'acteur ne trouve pas tout de suite ce qu'il a à jouer. Le masque, non. II doit vivre immédiatement, sans attendre, dès son entrée sur le plateau, dès son apparition. Le masque s'impose à l'acteur et impose ses lois. II est d'une souveraineté implacable. II ne se soumet à aucune autre logique que la sienne. C'est la raison pour laquelle l'improvisation libre est la forme qui lui est le plus appropriée, et que la rencontre avec un texte doit revêtir un caractère de nécessité.
Quand cette conjonction advient - entre l'acteur, le masque et le personnage tel qu'il apparaît dans les mots mêmes de l'auteur - cela peut être formidable. Mais si cette conjonction n'est pas parfaite, le texte ne s'entendra pas, car le masque imposera ses codes de jeu. Si un acteur, par exemple, veut travailler Puck dans Le Songe d'une Nuit d'Été, avec le masque de Polichinelle, Puck deviendra alors Polichinelle. Il en sera de même pour Figaro, Cyrano, Vladimir ou Estragon: Polichinelle dans tous les cas s'imposera. Le masque nivellera les différences de style d'écriture et d'enjeux dramatiques. Car le masque se sert plus d'un texte comme nourriture de jeu qu'il ne se met à son service.

Sous le masque, tout doit être montré au spectateur, objectivé en quelque sorte. Les formes extrêmes, telles que la farce ou la tragédie, gagnent à ce traitement car il y a dans leur écriture cette part de généralisation et d'essentialisation que demande le masque. Une écriture de type réaliste et psychologique, en revanche, sera étouffée par le recours au masque. Si l'on prend le cas de Tchekhov par exemple, la plupart de ses pièces en un acte résistent bien à cette mise à l'épreuve du masque qui viendra accentuer leur dimension farcesque, mais si l'on voulait faire l'expérience de travailler La Mouette avec des masques une fois dépassée la difficulté, immense, de trouver (ou fabriquer) des masques adéquats à ce travail -, on s'apercevrait vite de l'impossibilité qu'il y a à jouer ce que le travail de la langue fait déjà: masquer l'intention profonde par une phrase apparemment anodine. Le masque alors ne révèle rien. II brouille plutôt le jeu. Le travail accompli sur la langue par le poète fait déjà office de masque.

Le masque est en soi un texte. II contient 
son propre texte, car il est une partition précise comme peut l'être un rôle de théâtre. D'un autre côté, le texte agit comme un masque, car il met en lumière, ou dissimule, certaines pensées du personnage. Ces deux instruments sont très puissants pour l'acteur, car ils lui permettent d'aller dans des zones hors de son expérience habituelle. Mais, pour pouvoir les utiliser conjointement, il faut que leur complémentarité soit nécessaire, sinon leur pouvoir spécifique s'annule, et ils se desservent mutuellement.

Puisque le masque, pour faire entendre un texte, doit être en adéquation profonde avec celui-ci, une des difficultés réside dans l'éventail des masques que nous offrons aux acteurs dans le cadre d'une exploration scénique. Car si nous ne leur proposons que des masques de commedia dell'arte, par exemple, nous limitons fortement les résonances de certains textes. Autant, en effet, la tradition de la comédie italienne - de Ruzzante à Goldoni, en passant par Molière, Marivaux et les maîtres du théâtre de Foire - trouve un tremplin formidable dans ces retrouvailles avec les types traditionnels, tels Pantalon, Arlequin et autres masques de la commedia dell'arte, autant ces mêmes masques ne permettent pas une lecture ouverte, signifiante et inventive de textes plus contemporains. Trop de conventions sont contenus en eux et empêchent leur rayonnement. Il est bon alors de recourir à des masques moins chargés culturellement pour ouvrir l'imaginaire et le sens, et proposer, par exemple, des demi-masques balinais pour accentuer le style burlesque de certaines pièces de Brecht, qui viendront de surcroît apporter un effet d'"étrangéisation" pertinent et ludique ${ }^{27}$. D'où

27 Brecht demande ainsi dans une note de Combien coûte le fer : " Cette petite pièce doit être jouée dans le style burlesque du "knockabout" ", in Bertolt BRECHT, Théâtre complet 8, L'Arche, 1979, p. 134. l'importance de s'ouvrir à d'autres traditions extra-occidentales du théâtre masqué (et en particulier asiatique, si riche dans ce domaine) et de travailler en collaboration avec un facteur de masques afin qu'il puisse proposer de nouveaux types adaptés aux personnages et aux auteurs étudiés.

L'intérêt de ce type de confrontation entre masque et texte nous paraît double. Tout d'abord, elle permet de questionner le masque en tant qu'objet, et de repérer concrètement ses divers usages: du masque-cagoule au masque oriental, du demi-masque au masque entier articulé, du masque-prothèse au masque surdimensionné. D'autre part, en questionnant d'une manière inhabituelle le jeu, elle renouvelle l'approche interprétative du texte, en venant combiner la démarche intellective à la démarche organique. Le masque propose en effet toute une série de "déplacements" qui s'avèrent être des tremplins insoupçonnés pour l'acteur, ainsi que pour l'écriture scénique : décalage du visuel et du sonore, autonomie des gestes, importance des arrêts et des ruptures, musicalité renforcée des déplacements et du phrasé. La poétique du masque tient à tous ces déplacements, à ce devoir pour l'acteur d'aller dans les zones transfrontalières, pour déjouer les limites et inventer un théâtre poétique privilégiant l'imaginaire.

Recebido: 03/01/2017

Aprovado: 12/11/2017 